W1F-(5)-4

\title{
CHARACTERIZATION OF ULTRASHORT OPTICAL PULSES : A COMPARISON BETWEEN TOAD AND FROG
}

\author{
Tzu-Ming Liu, Yin-Chieh Huang, Gia-Wei Chern, Kung-Hsuan Lin, Chih-Jie Lee, Yu-Chueh \\ Hung, and Chi-Kuang Sun \\ Graduate Institute of Electro-Optical Engineering, National Taiwan University, Taipei 10617, \\ Taiwan, R.O.C. \\ Phone : 02-23635251ext.406, FAX : 02-23677467, E-mail : $\underline{8941002 @ e e . n t u . e d u . t w}$
}

Abstract -- Comparisons between second-harmonic-generation based frequency resolved optical gating (SHG FROG) and triple-optical autocorrelation for direct pulse-shape measurement (TOAD). have been made by computer simulation and experiments. Results show that TOAD has better accuracy than FROG.

\section{INTRODUCTION}

In the later 1960, it was shown that a triple-correlation is sufficient to determine the temporal intensity of a laser pulse with a direct mathematical calculation [1]. Direct third-harmonic-generation (THG) implementation of this proposal was realized on mode-locked $\mathrm{Cr}$ :forsterite and Ti:sapphire laser with the method of Triple-Optical Autocorrelation for Direct pulse-shape measurement (TOAD) [2]. In this report, we compare TOAD with commonly used method, FROG, by performing both methods on the same optical pulses. Accuracy analysis is also made by computer simulation. Results show that TOAD has better accuracy than FROG.

\section{ACCURACY COMPARISON AND RESULTS}

The iterative algorithm, which TOAD used, is Gerchberg-Saxton (GS) algorithm. With a measured spectrum and the pulse-shape calculated from triple-correlation [1], GS algorithm can retrieved the phase information of pulses [2]. To inspect the accuracy of the GS algorithm developed for TOAD, we compare it with the polarization gating FROG using computer simulation. For GS algorithm, the accuracy can be evaluated by

$$
\varepsilon_{G S}^{(k)} \equiv\left\{1 / N \sum_{i=1}^{i=N}\left[I_{G S}^{(k)}\left(\tau_{i}\right)-I_{T O A D}\left(\tau_{i}\right)\right]^{2}\right\}^{1 / 2}
$$

which is similar to E-field (root-mean-square) error for FROG. As an example, we use a squarish pulse with intensity I $(t)$ proportional to $\exp \left(-\mathrm{t}^{4}\right)$. Then we created the FROG trace for FROG algorithm and we created the intensity in both frequency and time domains for GS algorithm. Then we applied computer simulation on these traces with FROG and GS algorithm separately. Their corresponding accuracies with increasing iteration numbers are shown in Fig. 1. We find that GS algorithm guarantees error decay and the error reduces faster than the FROG algorithm. More comparisons about TOAD performance and algorithm accuracy will be discussed at the talk.

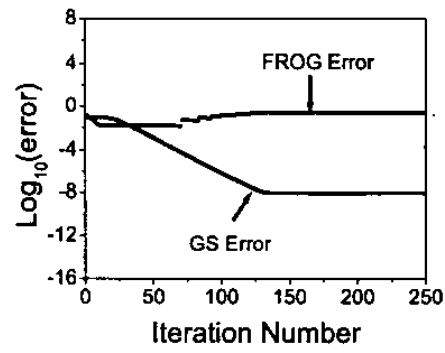

Fig. 1 Iteration errors, which resulted from computer simulation with FROG and GS algorithm.

\section{REFERENCES}

[1] E.I. Blount and J.R. Klauder "Recovery of laser intensity from correlation data," J. Appl. Phys., vol. 40, pp. 2874-2875 (1969).

[2] T.-M. Liu et al., "Triple-optical autocorrelation for direct optical pulse-shape measurement," Appl. Phys. Lett., vol. 81, pp. 1402-1404 (2002). 